\title{
Single nucleotide polymorphism of GSTP1 and pathological complete response in locally advanced rectal cancer patients treated with neoadjuvant concomitant radiochemotherapy
}

Luca Nicosia, MD¹, Giovanna Gentile, PhD², Chiara Reverberi, MD¹, Giuseppe Minniti, PhD², Maurizio Valeriani, MD1, Vitaliana de Sanctis, MD¹, Luca Marinelli, MD¹, Fabiola Cipolla², Ottavia de Luca ${ }^{4}$, Maurizio Simmaco, PhD², Mattia F. Osti, MD

'Department of Radiation Oncology, Sant'Andrea Hospital, Sapienza University of Rome, Rome; ${ }^{2}$ Department of Neuroscience, Mental Health and Sense Organs, Sapienza University of Rome, Rome; ${ }^{3}$ Department of Neurological Sciences, IRCCS Neuromed,

Pozzilli; ${ }^{4}$ Department of Advanced Molecular Diagnostic, Sant'Andrea Hospital, Sapienza University of Rome, Rome, Italy

Purpose: Standard treatment for locally advanced rectal cancer consists of neoadjuvant radiochemotherapy with concomitant fluoropyrimidine or oxaliplatin and surgery with curative intent. Pathological complete response has shown to be predictive for better outcome and survival; nevertheless there are no biological or genetic factors predictive for response to treatment. We explored the correlation between the single nucleotide polymorphisms (SNPs) GSTP1 (A313G) and XRCC1 (G28152A), and the pathological complete response and survival after neoadjuvant radiochemotherapy in locally advanced rectal cancer patients.

Materials and Methods: Genotypes GSTP1 (A313G) and XRCC1 (G28152A) were determined by pyrosequencing technology in 80 patients affected by locally advanced rectal cancer.

Results: The overall rate of pathological complete response in our study population was $18.75 \%$. Patients homozygous AA for GSTP1 (A313G) presented a rate of pathological complete response of 26.6\% as compared to $8.5 \%$ of the AG+GG population ( $p=$ 0.04). The heterozygous comparison (AA vs. AG) showed a significant difference in the rate of pathological complete response $(26.6 \%$ vs. $6.8 \% ; p=0.034)$. GSTP1 AA+AG patients presented a 5 - and 8 -year cancer-specific survival longer than GSTP1 GG patients (87.7\% and $83.3 \%$ vs. $44.4 \%$ and $44.4 \%$, respectively) ( $p=0.014)$. Overall survival showed only a trend toward significance in favor of the haplotypes GSTP1 AA+AG. No significant correlations were found for XRCC1 (G28152A).

Conclusion: Our results suggest that GSTP1 (A313G) may predict a higher rate of pathological complete response after neoadjuvant radiochemotherapy and a better outcome, and should be considered in a more extensive analysis with the aim of personalization of radiation treatment.

Keywords: Rectal cancer, Radiochemotherapy, Single nucleotide polymorphism, Prognostic factor, Tumor response, Genetic marker

\section{Introduction}

Locally advanced rectal cancer represent $33 \%$ of newly diagnosed rectal cancer with 5 -year survival of $69.5 \%$ [1]. Treatment of choice is represented by neoadjuvant radiochemotherapy followed by surgery, that allow to perform

Received 01 March 2018, Revised 31 March 2018, Accepted 14 June 2018.

Correspondence: Luca Nicosia, MD, Department of Radiation Oncology, Sant'Andrea Hospital, Sapienza University of Rome, via di Grottarossa 1035-39, Rome, Italy. Tel: +39-0633776160, Fax: +39-0633776608, E-mail: lucanicosia.rg@gmail.com

(c) This is an Open Access article distributed under the terms of the Creative Commons Attribution Non-Commercial License (http://creativecommons.org/ licenses/by-nc/4.0/) which permits unrestricted non-commercial use, distribution, and reproduction in any medium, provided the original work is properly cited.

www.e-roj.org 
a more conservative, sphincter saving, surgery, as compared to surgery alone [2-5].

Despite advances in neoadjuvant strategies local relapse occurs in 7\%-10\% of patients, especially in those who did not have a pathological complete response. Local relapse is in-field or marginal in 79\% of the cases, with 3- and 5-year overall survivals of $36.7 \%$, and $19.1 \%$, respectively $[6,7]$.

The pathological complete response has been proven to be correlated with a lower incidence of local recurrence and is predictive of longer survival [8]. Therefore, the identification of biological or genetic factors predictive to pathological complete response may help physicians in the personalization of the treatment. However, at the moment there are no proven factors routinely used in the clinical practice.

Radiotherapy applies its cytotoxic effects through the damage to cell structures, proteins, and DNA. Mechanisms of radiotherapy damage are essentially delivered indirectly, by reactive oxygen intermediate, and directly by damage to the cellular structures. Individual variations in the pathways of DNA repair and xenobiotic metabolism can be implicated in the mechanisms of radiosensitivity.

Recently, the correlation between genetic mutation and the development of side effects in rectal cancer patients has been investigated [9-13]. Mutations regarding genes involved in the regulation of cell cycle, apoptosis, and tumorigenesis, expression of growth factor receptors, nucleoside synthesis, and DNA repair has been considered and analyzed. The normal human genome presents numerous germinal variations in DNA sequences called polymorphisms. These variations are represented in more than 90\% of cases by single-nucleotide polymorphisms (SNPS) [14], some of which seem to be associated with the response to neoadjuvant radiochemotherapy in rectal cancer $[15,16]$.

The further examination of the genetic variation of the mechanisms of DNA repair may suggest in the future a potential benefit of intensified treatments in selected subgroups. Furthermore, inefficient treatments could be avoid in unfavourable candidates.

The aim of the study is to evaluate the role of the SNPS GSTP1 (glutathione S-transferase pi) A313G (rs1695) and XRCC1 (x-ray repair cross-complementing 1) G28152A ( $r$ 25487) as predictive markers of pathological complete response in locally advanced rectal cancer patients treated with neoadjuvant radiochemotherapy. Also overall survival and cancer-specific survival were assessed.

\section{Materials and Methods}

\section{Patients}

Between November 2008 and June 2015, 85 patients affected by locally advanced rectal cancer receiving neoadjuvant radiochemotherapy were included. At the final revision, complete data regarding preoperative and postoperative imaging, genetic assessment and histopathological evaluation were available for 80 patients that represent the study population. Patients' characteristics are summarized in Table 1.

Table 1. Patients' characteristics and clinical outcome $(n=80)$

\begin{tabular}{|c|c|}
\hline Characteristic & Value \\
\hline Age (yr) & $64(36-85)$ \\
\hline \multicolumn{2}{|l|}{ Sex } \\
\hline Male & $49(61.25)$ \\
\hline Female & 31 (38.75) \\
\hline \multicolumn{2}{|l|}{ T stage } \\
\hline T2 & $9(11.25)$ \\
\hline T3 & $67(83.75)$ \\
\hline T4 & $4(5.00)$ \\
\hline \multicolumn{2}{|l|}{ N Stage } \\
\hline No & $37(46.25)$ \\
\hline N1 & $32(40.00)$ \\
\hline N2 & $11(13.75)$ \\
\hline \multicolumn{2}{|c|}{ Distance from anal verge $(\mathrm{cm})$} \\
\hline$\leq 5$ & $44(55.00)$ \\
\hline$>5$ & $36(45.00)$ \\
\hline \multicolumn{2}{|l|}{ Surgery procedure } \\
\hline$\leq 5 \mathrm{~cm}$ & $69(86.25)$ \\
\hline RAR & $10(12.50)$ \\
\hline APR & $1(1.25)$ \\
\hline TAE & - \\
\hline \multicolumn{2}{|l|}{ урт stage } \\
\hline T0 & $15(18.75)$ \\
\hline $\mathrm{T} 1$ & $4(5.00)$ \\
\hline T2 & $22(27.50)$ \\
\hline T3 & $38(47.50)$ \\
\hline T4 & $1(1.25)$ \\
\hline \multicolumn{2}{|l|}{ ypN stage } \\
\hline No & $60(75.00)$ \\
\hline N1 & $16(20.00)$ \\
\hline N2 & $4(5.00)$ \\
\hline \multicolumn{2}{|l|}{ Dworak TRG sec. } \\
\hline 0 & $7(8.75)$ \\
\hline 1 & $17(21.25)$ \\
\hline 2 & $28(35.00)$ \\
\hline 3 & 13(16.25) \\
\hline 4 & 15(18.75) \\
\hline
\end{tabular}

Values are presented as median (range) or number (\%). $A R$, rectum anterior resection; $A P R$, abdominoperineal resection; $T A E$, transanal excision; TRG, tumour regression score. 
Inclusion criteria were: age $\geq 18$ years, biopsy-proven diagnosis of adenocarcinoma localized $\leq 10 \mathrm{~cm}$ from the anal verge, locally advanced rectal cancer (stage II-III), complete staging including colonoscopy, contrast enhanced multi parametric-MRI of the pelvis and total body CT. Exclusion criteria were: evidence of distant metastases, inability to receive radiotherapy associated to chemotherapy.

\section{Treatment}

The radiation treatment was delivered using a threedimensional conformal technique. The clinical target volume 1 (CTV1) included the entire macroscopic tumour, the mesorectum, internal iliac and presacral lymph nodes, adding a margin of $1 \mathrm{~cm}$ in all directions to generate the corresponding planning treatment volume 1 (PTV1). The boost volume (CTV2) included the macroscopic tumour and the corresponding mesorectum for $1-1.5 \mathrm{~cm}$ in craniocaudal direction from the external margin of the tumor. Subsequently, a margin of $1 \mathrm{~cm}$ in all directions was added to generate the PTV2. The total prescribed dose to the PTV1 was 45 Gy delivered in 25 fractions of 1.8 Gy per day for 5 days a week over 5 weeks, plus a concomitant boost of $10 \mathrm{~Gy}$ in 10 fractions delivered biweekly to the PTV2 (total dose, 55 Gy).

All patients received concurrent chemotherapy: 48 patients (60\%) received capecitabine $(825 \mathrm{mg} / \mathrm{mq})$ twice daily and 32 patients (40\%) received a daily continuous infusion of 5 -fluorouracil (225 mg/mq/day) on each day of radiotherapy. Contrast enhanced multi parametric-MRI of the pelvis was performed for each patient to evaluate the tumour response after 4 weeks of neoadjuvant radiochemotherapy. A sphinctersaving surgery was evaluated taking into account the response to combined treatment and the distal from anal verge, and was performed 6-8 weeks after neoadjuvant radiochemotherapy. Sixty-nine patients (86.25\%) received an anterior resection, 10 patients (12.5\%) an abdominoperineal resection, and 1 patient (1.25\%) a transanal endoscopic microsurgery. Adjuvant chemotherapy was administered in 25 patients (31.25\%), 16
(64\%) received FOLFOX, and 9 (36\%) XELOX.

\section{Gene selection and genotype analysis}

After a careful review of the literature candidate SNPS GSTP1 A313G (rs1695) and XRCC1 G28152A (rs25487), were chosen on the basis of a radiation-related mechanism of action and previous studies that included large population or that were perspective $[15,16]$. Genes directly linked to the metabolism of fluoropyrimidines were not chosen to avoid possible confounding factors.

Blood sample was collected before the start of treatment and genomic DNA was isolated using the X-tractor Gene system (Corbett Life Science, Sydney, Australia). GSTP1 A313G (rs1695) and XRCC1 G28152A (rs25487) were examined. Reference sequences for the genes were obtained from NCBI GenBank database (http://www.ncbi.nlm.nih.gov/). The analyzed SNPs were chosen based on previous evidence and the high prevalence in white population. The Hardy-Weinberg equilibrium was fulfilled for the genotyped sites ( $p>0.1, \chi^{2}$ test) and the prevalence of alleles had similar distribution compared with the Caucasian population, as shown in Table 2. Genotyping was performed by pyrosequencing technology, using the pyrosequencer (PyroMark ID system; Qiagen, Hilden, Germany) according to manufacturers' instructions. Both the amplification and the sequencing primers were obtained by the PSO Assay Design software (Biotage AB and Biosystems Uppsala, Sweden). Sequence of selected primers is reported in Table 3. The region covering the SNPs of interest was amplified by polymerase chain reaction (PCR). The PCR requirements were: $95^{\circ} \mathrm{C}$ for 3 minutes; 40 cycles with denaturation at $95^{\circ} \mathrm{C}$ for 30 seconds, annealing at $56^{\circ} \mathrm{C}$ for 30 seconds, and elongation at $72^{\circ} \mathrm{C}$ for 30 seconds; a final extension step at $72^{\circ} \mathrm{C}$ for 5 minutes. The PCRs were performed in a final volume of $25 \mu \mathrm{L}$ (except for GSTP1, for which $50 \mu \mathrm{L}$ were used), containing $70 \mathrm{ng}$ of genomic DNA, 10 pmol of primers, $0.2 \mathrm{mM}$ dNTPs, PCR buffer, $1 \mathrm{mM} \mathrm{MgCl}$, and $1 \mathrm{U}$ of Taq DNA polymerase (Takara Bio Inc., Otsu, Japan).

Table 2. Localization and observed frequency of the analyzed SNPS

\begin{tabular}{lccccc}
\hline \multicolumn{1}{c}{ SNP } & $\begin{array}{c}\text { Chromosomal } \\
\text { localization }^{\text {a) }}\end{array}$ & Amino acid position & Genotype $^{\text {b) }}$ & $\begin{array}{c}\text { No. of } \\
\text { patients }\end{array}$ & $\begin{array}{c}\text { MAF expected } \\
\text { (observed) }\end{array}$ \\
\hline GSTP1 A313G (rs1695) & $11: 67585218$ & Codon 105-313 & A/G & 80 & 0.33 (0.3) \\
XRCC1 G28152A (rs25487) & $19: 43551574$ & Exon 10-399 & G/A & 80 & $0.34-0.36(0.38)$ \\
\hline
\end{tabular}

SNP, single nucleotide polymorphisms; MAF, minor allele frequency.

${ }^{a)}$ Chromosome indicates position. ${ }^{\text {b) }}$ Genotype nomenclature refers to the DNA sense strand. 
Table 3. Primers for PCR amplification and pyrosequencing

\begin{tabular}{lccc}
\hline \multicolumn{1}{c}{ SNP } & Forward primer $\left(5^{\prime}-3^{\prime}\right)$ & Reverse primer $\left(5^{\prime}-3^{\prime}\right)$ & Sequencing primer (5'-3') \\
\hline GSTP1 A313G (rs1695) & ${ }^{a}$ GTGGACATGGTGAATGAC & GCTCACATAGTTGGTGTAGA & GTTGGTGTAGATGAGGG \\
XRCC1 G28152A (rs25487) & ${ }^{a}$ AGTACAGCCAGGTCCTAG & CGCTCCTCTCAGTAGTCT & CGTGTGAGGCCTTACC \\
\hline
\end{tabular}

Primers are tagged with a biotin molecule attached.

$\mathrm{PCR}$, polymerase chain reaction.

\section{Follow-up and statistics}

Pathologic response of the tumours was assessed by postoperative histopathological evaluation, according to the tumour regression grade (TRG) by Dworak Scale [17]. The tumour response in our series was therefore classified as follow: pathological complete response (TRG 4), no tumour cells, only fibrotic mass; major pathological response (TRG 3), very few tumour cells in fibrotic tissue with or without mucous substance; good pathological response (TRG 2), dominant fibrotic change with few poor tumour cells or groups; poor pathological response (TRG 1), dominant tumour mass with obvious fibrosis and/or vasculopathy; and absent pathological response (TRG 0), no regression.

Patients were classified into three groups on the basis of the allelic assessment (normal homozygous, mutated homozygous, heterozygous). Each group was then divided as non-complete responder (TRG 0-3) or complete responder (TRG 4). A univariate model using a $\chi^{2}$ test (in $2 \times 2$ contingency tables) was performed in different genetic comparison models, including homozygote comparison (AA vs. aa), heterozygote comparison (Aa vs. aa; Aa vs. AA), dominant model (aa vs. $A a / A A)$, and recessive model ( $A A$ vs. Aa/AA). Overall survival and cancer-specific survival were estimated for patients with $\geq 12$ months of available follow-up using the Kaplan-Meier method. Prognostic factors such as age, sex, clinical stage, pathological T and $\mathrm{N}$, type of surgery, adjuvant chemotherapy, TRG and SNPs assessment were included in the univariate analysis using the log-rank test. Cox regression method and multiple logistic regression were used in multivariate analysis. Statistical analysis was performed using the SPSS statistical software package version 22.0 (IBM, Armonk, NY, USA). A p s 0.05 indicated a significant association.

\section{Results}

1. Treatment outcome and association to GSTP1 (A313G) Fifteen patients (18.75\%) had a pathological complete response: 12 (80\%) of them were homozygous AA, 2 (13.5\%) were heterozygous $A G$, and 1 (6.5\%) was homozygous GG.
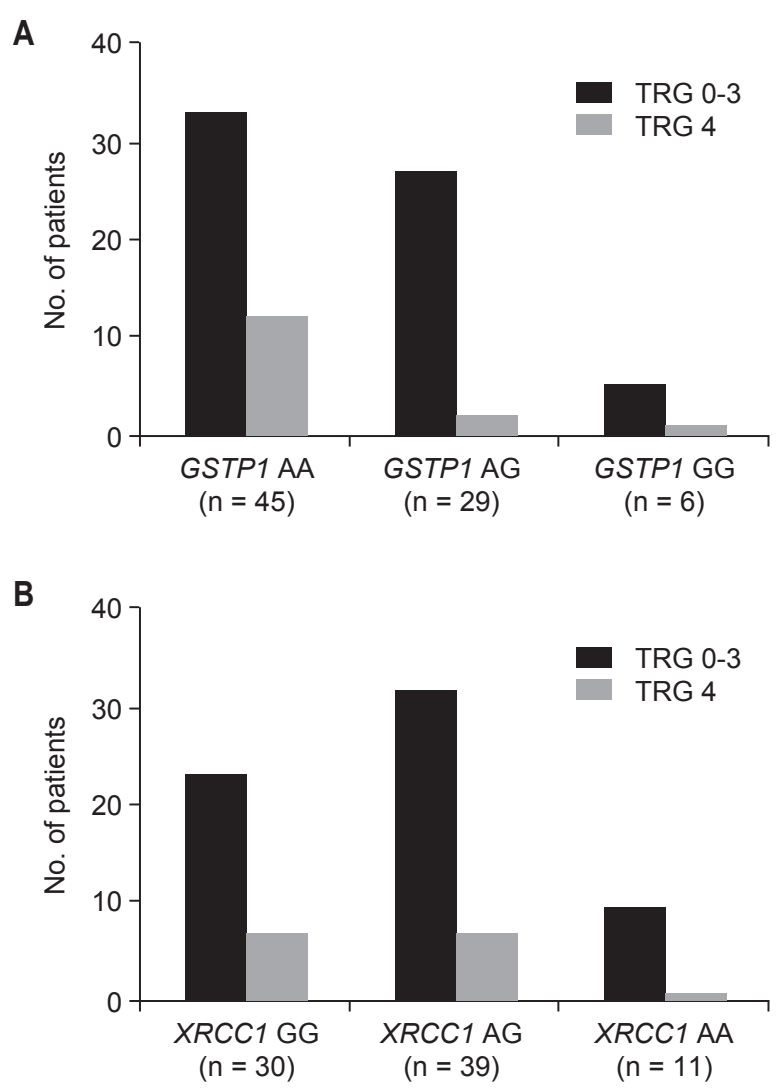

Fig. 1. Single nucleotide polymorphisms characteristics and response to treatment according to allelic assessment $(n=80)$. GSTP1, glutathione S-transferase pi; XRCC1, X-ray repair crosscomplementing $1 ; \mathrm{TRG}$, tumour regression grade.

The distribution of the pathological response in our study population is reported in Fig. 1A.

Among the patients homozygous $A A$ we observed a pathological complete response rate of $26.6 \%$ while in the population $A G+G G$ the rate was $8.5 \%(p=0.04)$. The heterozygote comparison ( $A A$ vs. $A G$ ) showed a significant difference in the rate of pathological complete response (26.6\% vs. $6.8 \% ; p=0.034$ ). No correlations have been found between GSTP1 (A313G) and downstaging of primary tumor or lymph nodes. 


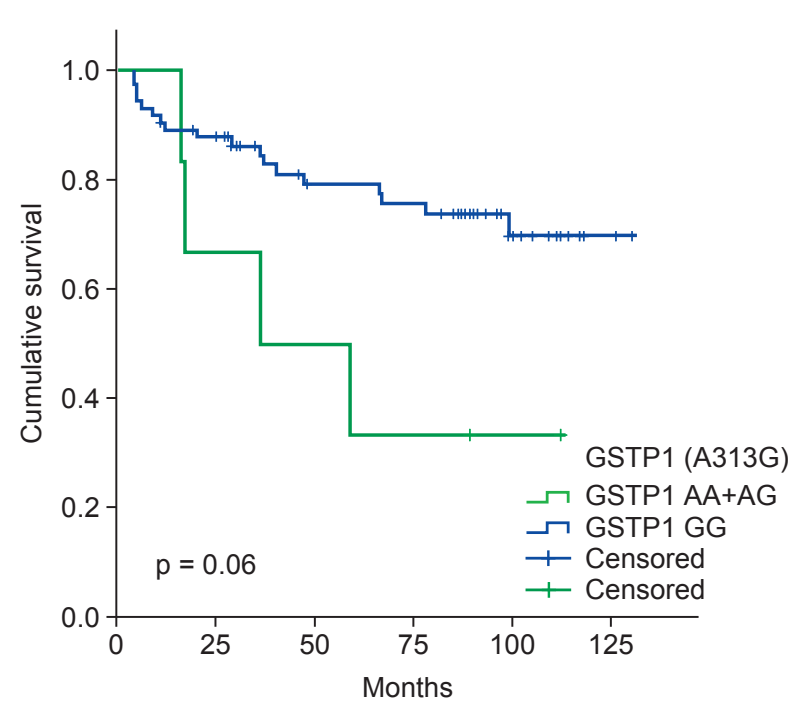

Fig. 2. Overall survival stratified for GSTP1 AA+AG vs. GG.

The median overall survival was not reached; actuarial overall survival is 73.3\%. The 5- and 8-year overall survivals stratified for AA vs. AG vs. GG were $72.9 \%$ and $61.1 \%, 76.9 \%$ and $76.9 \%$, and $33.3 \%$ and $33.3 \%$, respectively ( $p=0.075$ ). When compared $G G$ vs. $A A+A G$ the difference in overall survival showed a trend towards significance with rates at 5 - and 8 -year of $33.3 \%$ and $33.3 \%, 74.6 \%$ and $66.8 \%$, respectively ( $p=0.06$ ) (Fig. 2 ).

Median cancer-specific survival was not reached; actuarial cancer-specific survival is 84\%. The 5- and 8-year cancerspecific survivals stratified for AA vs. AG vs. GG were: $89.1 \%$ and $82 \%, 85.1 \%$ and $85.1 \%, 44.4 \%$ and $44.4 \%$, respectively. A significant difference between groups was found ( $p=0.048$ ). The cancer-specific survivals between the haplotype $G G$ and the combined population $A A+A G$ at 5 and 8 years was $44.4 \%$ and $44.4 \%, 87.7 \%$ and $83.3 \%$, respectively $(p=0.014)$ (Fig. 3 ).

\section{Treatment outcome and association to XRCC1 (G28152A)}

The rate of pathological complete response for the haplotypes $A A, A G$, and $G G$ was 9\%, 17.9\%, and 23.3\%, respectively (Fig. $1 B$ ). This difference was not statistically significant in all the analyzed models.

The 5- and 8-year overall survivals for the haplotypes of XRCC1 (G28152A) was as follows: GG (69.5\% and 54.8\%), AG (76.3\% and 76.3\%), and AA (45\% and 45\%) ( $p=0.145)$. The 5and 8-year cancer-specific survivals stratified for haplotypes were as follows: GG (82.7\% and 77.2\%), AG (90.7\% and $90.7 \%)$, and $A A(52.5 \%$ and $52.5 \%)(p=0.072)$.

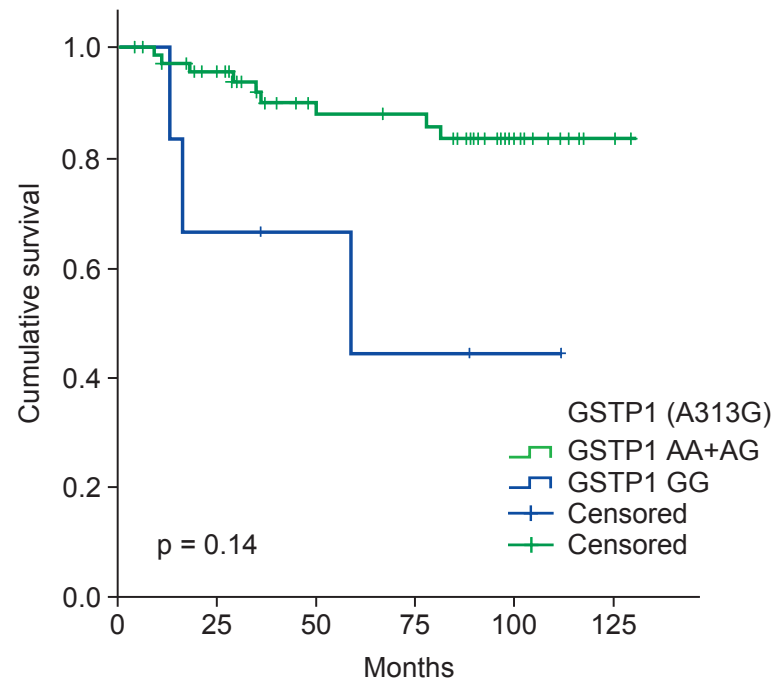

Fig. 3. Cancer-specific survival stratified for GSTP1AA+AG vs. GG.

No correlations have been found between XRCC1 (G28152A) and down-staging of primary tumor and lymph nodes.

\section{Pattern of relapse}

After a median follow-up of 83 months (range, 4 to 130 months), local relapse occurred in 8 patients (10\%), regional relapse occurred in 1 patient (1.25\%; external iliac lymph nodes), and systemic relapse in 20 patients (25\%). Seventy-five patients (93.75\%) were evaluated for survival.

Systemic relapse occurred more frequently in patients homozygous GG for GSTP1, as compared to AA and AG (83.3\%, $23.3 \%$, and $21.4 \%$, respectively; $p=0.006$ ) and in patients with yN2 nodal status as compared to yN1 and yNo (66.7\%, $46.7 \%$, and $20.3 \%$, respectively; $p=0.036$ ). GSTP1 GG was not correlated with higher rate of yN2 nodal status.

\section{Prognostic factors}

At the univariate analysis, the presence of the gene G of GSTP1 (A313G) correlated significantly with a lower overall survival ( $p$ $=0.046)$; moreover pathological nodal status $(y N)(p=0.013)$ and GSTP1 GG ( $p=0.028$ ) were significantly correlated with a lower cancer-specific survival. At the multivariate analysis, GSTP1 GG ( $p=0.039$; HR = 0.27; confidence interval, 0.12-0.31) was a significant factor associated with a lower cancer-specific survival, but not with overall survival. Moreover GSTP1 GG significantly correlated with a lower incidence of pathological complete response $(p=0.049$; $O R=0.2$; confidence interval, 0.42-0.99) (Tables 4, 5). 
Table 4. Multivariate analysis: overall survival vs. cancer-specific survical

\begin{tabular}{|c|c|c|c|c|c|c|}
\hline \multirow{2}{*}{ Factor } & \multicolumn{3}{|c|}{ Overall survival } & \multicolumn{3}{|c|}{ Cancer-specific survival } \\
\hline & $H R$ & $\mathrm{Cl}$ & $p$-value & $\mathrm{HR}$ & $\mathrm{Cl}$ & $p$-value \\
\hline Age ( $\geq 65 \mathrm{yr}$ ) & 1.07 & $0.95-1.03$ & 0.873 & 0.28 & $0.07-0.11$ & 0.072 \\
\hline Sex & 0.97 & $0.43-2.19$ & 0.951 & 1.16 & $0.33-4.09$ & 0.812 \\
\hline $\mathrm{N}$ down-staging & 0.85 & $0.36-1.98$ & 0.718 & 0.34 & $0.08-1.36$ & 0.128 \\
\hline GSTP1 GG & 0.44 & $0.14-1.4$ & 0.178 & 0.27 & $0.12-0.31$ & 0.039 \\
\hline TRG 4 & 0.50 & $0.1-2.94$ & 0.15 & 0.70 & $0.62-1.13$ & 0.6 \\
\hline
\end{tabular}

$\mathrm{HR}$, hazard ratio; $\mathrm{Cl}$, confidence interval; GSTP1, glutathione $\mathrm{S}$-transferase pi; TRG, tumour regression grade.

Table 5. Multivariate analysis of TRG 4

\begin{tabular}{lccc}
\hline \multirow{2}{*}{ Factor } & \multicolumn{3}{c}{ TRG 4} \\
\cline { 2 - 4 } & OR & Cl & p-value \\
\hline GSTP1 AA & 0.37 & $0.20-4.90$ & 0.451 \\
GSTP1 GG & 0.20 & $0.42-0.99$ & 0.049 \\
\hline
\end{tabular}

$T R G$, tumour regression grade; $O R$, odds ratio; $\mathrm{Cl}$, confidence interval; GSTP1, glutathione S-transferase pi.

\section{Discussion and Conclusion}

The gold standard for the treatment of locally advanced rectal cancer in Western countries is neoadjuvant radiochemotherapy followed by surgery with curative intent. Many studies have evaluated clinical, pathological and molecular factors to predict for the prognosis of rectal cancer after neoadjuvant radiochemotherapy. Nevertheless, the histopathological classification of the surgical specimen remains the main parameter to define patients' prognosis. Current available data are not still sufficient to modify standard therapies into individualized treatments. Intensified regimens of radiochemotherapy could lead to a substantial increase in side effects and may be considered only for patients with very advanced stage or at high risk of local/distant relapse. On the other hand, inadequate therapy may worsen the outcome and can be considered only in very early stage disease.

We hypothesized that modifications in the system of reactive oxygen intermediate detoxification and in the DNA repair mechanism may lead to an increased efficacy of radiotherapy that can be translated in an increased response to treatment. In the present study, the correlation between the SNPs GSTP1 (A313G) and XRCC1 (G28152A) and pathological response, survival and pattern of relapse in locally advanced rectal cancer patients treated with neoadjuvant radiochemotherapy was analyzed.

GSTP1 is a polymorphic gene that encodes for a phase
Il enzyme involved in detoxification of carcinogenic agents [18]. The over-expression of GSTP1 protein has been reported in many solid and haematological human cancers such as breast cancer $[19,20]$, multiple myeloma and Monoclonal gammopathy of undetermined significance [21]. The polymorphism A313G leads to a substitution of an isoleucine with a valine in the codon 105 (lle105Val). This amino acid is located near the hydrophobic binding site for the electrophile substrate; thus, the protein has an altered affinity with the electrophile substrate and therefore a reduced catalytic activity [22], while the allele $G$ confers a higher catalytic efficiency for polycyclic aromatic hydrocarbon diol epoxides [23]. The demonstration of differential effects of the two alleles is therefore translated in a differential outcome for heterozygous gene (GA), as compared to the homozygous. The correlation between GSTP1 (A313G) and a higher predisposition to develop rectal cancer has been reported $[24,25]$, as well as a correlation with an intensified radiochemotherapy and toxicity $[13,26]$. The results regarding a possible correlation between GSTP1 (A313G) and the outcome after radiotherapy or radiochemotherapy in many tumours are controversial [27-30]. Lastly polymorphisms of GSTP gene have already been studied in rectal cancer with promising but still conflicting results.

In an extensive pharmacogenomic analysis on a subgroup of patients of the INT-0144 trial, Bohanes et al. [15] analyzed 18 polymorphisms in 16 gene finding a significant association between GSTP1 (A313G) and overall survival and disease-free survival. Patients with the genotypes AG and AA had higher 3and 5 -year disease-free survivals and overall survivals than the low activity genotype GG in a range of 5\%-12\% and 4\%-13\% for the disease-free survival, 1\%-5\% and 8\%-14\% for the overall survival, respectively.

In the present study, a significantly higher rate of pathological complete response was shown for AA genotype compared to other genotypes ( $A G$ and $G G$ ). Lower survival of GG haplotype patients was found when compared to the 
$A A+A G$ patients. After 83 months of median follow-up, 5and 8-year cancer-specific survivals were significantly better for $A A+A G$ patients as compared to $G G$ patients; while overall survivals had only a trend towards significance.

The results of the two studies, highlights the importance of rate of response to treatment, according to genetic parameters as a key step in the way of the personalization of treatment, due to the reported correlation between pathological complete response and longer survival [8].

Cecchin et al. [31] analyzed 25 polymorphisms in 16 genes in a population of rectal cancer patients treated with neoadjuvant radiochemotherapy, fluoropyrimidine alone or in combination and reported no association between GSTP1 (A313G) and the TRG. Different treatment regimens, histopathological evaluation (Dworak scale vs. Mandard criteria) and outcome measurements may have contributed to these inconsistencies with our results.

$X R C C 1$ is one of the most important genes of the base excision repair (BER) pathway. The encoded protein interacts with the DNA polymerase and the DNA ligase III in response to the damage of single DNA strand caused by the interaction with ionizing radiations, reactive oxygen intermediate and methylating agents. The substitution of a guanine with an adenine in position 28152 (G28152A) in exon 10 of the gene encoding for XRCC1 leads to a substitution of an arginine with a glutamine in the codon 399 (Arg399GIn) and it is associated with a lower DNA repair ability [32,33].

In our series, no correlation between XRCC1 (G28152A) and the rate of pathological complete response and survival has been found. Our results are in accordance to the findings by Balboa et al. [34], where the mutation was not able to predict the response after neoadjuvant radiochemotherapy with fluoropyrimidine-based chemotherapy in locally advanced rectal cancer patients. In contrast, Paez et al. [16] analyzed 9 SNPs in 128 rectal cancer patients, showing that XRCC1 (G28152A) was predictive for a longer progression-free survival. Interestingly, the other analyzed SNPs including GSTP1 (A313G) were not correlated to pathological stage and survival. These results could reflect the complexity of genes interaction.

Our study present some limits such as the retrospective nature and the small study population. On the other hand, our series underwent homogeneous treatment and the results were analyzed after long-term follow-up. Furthermore, our patients were not treated with oxaliplatin, used in some trials in combination with fluoropyrimidine, a possible confounding factor due to the known metabolic correlation with GST-genes family $[26,35]$.

In conclusion, more attention should be paid to this new approach of personalized radiotherapy in the future. Further trials are needed to evaluate new combinations of treatment, or the effect of a different total dose or altered fractionation on the basis of the genetic mutations.

The long-term results of our study suggest the possibility to use GSTP1 (A313G) SNP to identify patients with different sensitivity to neoadjuvant radiochemotherapy. Mutated gene predicted for low rate of pathological complete response and poor survival. Future studies should confirm these results in a larger cohort of patients and evaluate the effects of an intensification of treatment in patients more sensitive to radiations. The upcoming evidence supporting the genetic basis of the radiosensitivity should arise the interest in deepen the study of these mechanisms with the aim of a personalized radiotherapy.

\section{Conflict of Interest}

No potential conflict of interest relevant to this article was reported.

\section{References}

1. Siegel $R$, Desantis $C$, Jemal A. Colorectal cancer statistics, 2014. CA Cancer J Clin 2014;64:104-17.

2. Janjan NA, Khoo VS, Abbruzzese J, et al. Tumor downstaging and sphincter preservation with preoperative chemoradiation in locally advanced rectal cancer: the M. D. Anderson Cancer Center experience. Int J Radiat Oncol Biol Phys 1999;44:102738.

3. Kim JS, Kim JS, Cho MJ, Song KS, Yoon WH. Preoperative chemoradiation using oral capecitabine in locally advanced rectal cancer. Int J Radiat Oncol Biol Phys 2002;54:403-8.

4. Bartelink H, Roelofsen F, Eschwege $F$, et al. Concomitant radiotherapy and chemotherapy is superior to radiotherapy alone in the treatment of locally advanced anal cancer: results of a phase III randomized trial of the European Organization for Research and Treatment of Cancer Radiotherapy and Gastrointestinal Cooperative Groups. J Clin Oncol 1997;15:2040-9.

5. Rodel C, Graeven U, Fietkau R, et al. Oxaliplatin added to fluorouracil-based preoperative chemoradiotherapy and postoperative chemotherapy of locally advanced rectal cancer (the German CAO/ARO/AIO-04 study): final results of the multicentre, open-label, randomised, phase 3 trial. Lancet 
Oncol 2015;16:979-89.

6. Bosset JF, Collette L, Calais G, et al. Chemotherapy with preoperative radiotherapy in rectal cancer. $\mathrm{N}$ Engl J Med 2006;355:1114-23.

7. Bujko K, Nowacki MP, Nasierowska-Guttmejer A, Michalski W, Bebenek M, Kryj M. Long-term results of a randomized trial comparing preoperative short-course radiotherapy with preoperative conventionally fractionated chemoradiation for rectal cancer. Br J Surg 2006;93:1215-23.

8. Maas M, Nelemans PJ, Valentini $V$, et al. Long-term outcome in patients with a pathological complete response after chemoradiation for rectal cancer: a pooled analysis of individual patient data. Lancet Oncol 2010;11:835-44.

9. Smith JJ, Wasserman I, Milgrom SA, et al. Single nucleotide polymorphism TGF $\beta 1$ R25P correlates with acute toxicity during neoadjuvant chemoradiotherapy in rectal cancer patients. Int J Radiat Oncol Biol Phys 2017;97:924-30.

10. Borchiellini D, Etienne-Grimaldi MC, Thariat J, Milano G. The impact of pharmacogenetics on radiation therapy outcome in cancer patients: a focus on DNA damage response genes. Cancer Treat Rev 2012;38:737-59.

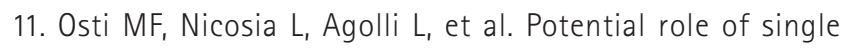
nucleotide polymorphisms of XRCC1, XRCC3, and RAD51 in predicting acute toxicity in rectal cancer patients treated with preoperative radiochemotherapy. Am J Clin Oncol 2017:40:535-42.

12. Zhang $H$, Wang M, Shi T, et al. TNF rs1799964 as a predictive factor of acute toxicities in Chinese rectal cancer patients treated with chemoradiotherapy. Medicine (Baltimore) 2015:94:e1955.

13. Agostini M, Pasetto LM, Pucciarelli $S$, et al. Glutathione S-transferase P1 Ile105Val polymorphism is associated with haematological toxicity in elderly rectal cancer patients receiving preoperative chemoradiotherapy. Drugs Aging 2008;25:531-9.

14. Mandola MV, Stoehlmacher J, Muller-Weeks S, et al. A novel single nucleotide polymorphism within the $5^{\prime}$ tandem repeat polymorphism of the thymidylate synthase gene abolishes USF-1 binding and alters transcriptional activity. Cancer Res 2003;63:2898-904.

15. Bohanes $P$, Rankin CJ, Blanke CD, et al. Pharmacogenetic analysis of INT 0144 Trial: association of polymorphisms with survival and toxicity in rectal cancer patients treated with 5-FU and radiation. Clin Cancer Res 2015;21:1583-90.

16. Paez D, Salazar J, Pare L, et al. Pharmacogenetic study in rectal cancer patients treated with preoperative chemoradiotherapy: polymorphisms in thymidylate synthase, epidermal growth factor receptor, GSTP1, and DNA repair genes. Int J Radiat Oncol Biol Phys 2011;81:1319-27.

17. Losi L, Luppi G, Gavioli M, et al. Prognostic value of Dworak grade of regression (GR) in patients with rectal carcinoma treated with preoperative radiochemotherapy. Int J Colorectal Dis 2006;21:645-51.

18. Harries LW, Stubbins MJ, Forman D, Howard GC, Wolf CR. Identification of genetic polymorphisms at the glutathione S-transferase Pi locus and association with susceptibility to bladder, testicular and prostate cancer. Carcinogenesis 1997;18:641-4.

19. Damaraju S, Murray D, Dufour J, et al. Association of DNA repair and steroid metabolism gene polymorphisms with clinical late toxicity in patients treated with conformal radiotherapy for prostate cancer. Clin Cancer Res 2006;12:2545-54.

20. Edvardsen H, Kristensen VN, Grenaker Alnaes GI, et al. Germline glutathione S-transferase variants in breast cancer: relation to diagnosis and cutaneous long-term adverse effects after two fractionation patterns of radiotherapy. Int J Radiat Oncol Biol Phys 2007;67:1163-71.

21. Di Simone D, Galimberti S, Mattii L, Petrini M. c-Jun and GST-pi expression in human plasma cells. Haematologica 1997:82:69-70.

22. Srivastava SK, Singhal SS, Hu $X$, Awasthi YC, Zimniak $P$, Singh SV. Differential catalytic efficiency of allelic variants of human glutathione S-transferase $\mathrm{Pi}$ in catalyzing the glutathione conjugation of thiotepa. Arch Biochem Biophys 1999;366:8994.

23. Watson MA, Stewart RK, Smith GB, Massey TE, Bell DA. Human glutathione S-transferase P1 polymorphisms: relationship to lung tissue enzyme activity and population frequency distribution. Carcinogenesis 1998;19:275-80.

24. Wang J, Jiang J, Zhao Y, et al. Genetic polymorphisms of glutathione S-transferase genes and susceptibility to colorectal cancer: a case-control study in an Indian population. Cancer Epidemiol 2011;35:66-72.

25. Ates NA, Tamer $L$, Ates $C$, et al. Glutathione S-transferase $M 1, T 1, P 1$ genotypes and risk for development of colorectal cancer. Biochem Genet 2005;43:149-63.

26. Kitade H, Shimasaki T, Igarashi $S$, et al. Long-term administration and efficacy of oxaliplatin with no neurotoxicity in a patient with rectal cancer: association between neurotoxicity and the GSTP1 polymorphism. Oncol Lett 2014;7:1499-502.

27. Abbas M, Kushwaha VS, Srivastava K, Banerjee M. Glutathione S-transferase gene polymorphisms and treatment outcome in 
cervical cancer patients under concomitant chemoradiation. PLoS One 2015;10:e0142501.

28. Okcu MF, Selvan M, Wang LE, et al. Glutathione S-transferase polymorphisms and survival in primary malignant glioma. Clin Cancer Res 2004;10:2618-25.

29. Sweeney C, Nazar-Stewart V, Stapleton PL, Eaton DL, Vaughan TL. Glutathione S-transferase M1, T1, and P1 polymorphisms and survival among lung cancer patients. Cancer Epidemiol Biomarkers Prev 2003;12:527-33.

30. Mahimkar MB, Samant TA, Kannan S, Tulsulkar J, Pai PS, Anantharaman D. Polymorphisms in GSTM1 and XPD genes predict clinical outcome in advanced oral cancer patients treated with postoperative radiotherapy. Mol Carcinog 2012;51 Suppl 1:E94-103.

31. Cecchin E, Agostini M, Pucciarelli $S$, et al. Tumor response is predicted by patient genetic profile in rectal cancer patients treated with neo-adjuvant chemo-radiotherapy.
Pharmacogenomics J 2011;11:214-26.

32. Au WW, Salama SA, Sierra-Torres CH. Functional characterization of polymorphisms in DNA repair genes using cytogenetic challenge assays. Environ Health Perspect 2003;111:1843-50.

33. Wang $Y$, Spitz MR, Zhu Y, Dong $Q$, Shete $S$, Wu X. From genotype to phenotype: correlating XRCC1 polymorphisms with mutagen sensitivity. DNA Repair (Amst) 2003;2:901-8.

34. Balboa E, Duran G, Lamas MJ, et al. Pharmacogenetic analysis in neoadjuvant chemoradiation for rectal cancer: high incidence of somatic mutations and their relation with response. Pharmacogenomics 2010;11:747-61.

35. Kim DY, Paek TY, Oh SY, et al. Pretreatment selection of regimen according to genetic analysis improves the efficacy of chemotherapy in the first line treatment of metastatic colorectal cancer. J Surg Oncol 2014;109:250-4. 